\title{
Ocuserts: A Novel Formulation Approach in Drug Delivery System
}

\author{
Nida Parveen ${ }^{1 *}$, Himanshu Joshi ${ }^{2}$
}

${ }^{1}$ Department of Pharmacy, Shri Ram Murti Smarak College of Engineering and Technology, Bareilly, U. P, India

${ }^{2}$ Invertis Institute of Pharmacy, Invertis University, Bareilly, U. P, India

\author{
DOI: $10.36348 /$ sjmps.2020.v06i05.005
}

| Received: 04.05.2020 | Accepted: 16.05.2020 | Published: 23.05.2020

*Corresponding author: Nida Parveen

\section{Abstract}

Eye is the most fantastic creation of God among all the sense organs in human body as it makes us aware of various objects all around us near and far. The eye is one of the most soft and most valuable of all the sense organs and is a challenging subject for topical administration of drug to the eye. Topical application of drug to the eye is the well accepted route of administration for the treatment of various eye disease like conjunctiva, eye flu etc. The eye as a portal for drug delivery is generally used for local therapy in order to avoid the risk of eye damage from high blood concentration of the drug which is not intended. The field of ocular drug delivery system is one of the most interesting and challenging task usually faced by pharmaceutical scientist/researchers. This field has significantly improved over the past 10-20 years.

Keywords: Ocuserts, Ophthalmic Dosage Forms, Drug Delivery into Eyes, Drug Release.

Copyright @ 2020: This is an open-access article distributed under the terms of the Creative Commons Attribution license which permits unrestricted use, distribution, and reproduction in any medium for non-commercial use (NonCommercial, or CC-BY-NC) provided the original author and source are credited.

\section{INTRODUCTION}

Ocusert system was firstly developed in 1975 by 'Alza Corporation, in the United State of America. It is a flat, flexible, solid and semisolid device which consists of drug reservoir and rate controlling membrane by using various polymers $[1,2]$. The prime objective of development of the ocuserts is continuous controlled delivery of ophthalmically active drug to the eye. The ocusert is inserted in the upper or lower culde-sac of the eye, which releases the drug at a predetermined rate constant. Thus improved patient compliance by reduced dose frequency, better therapeutic outcomes by reduced over/under dosing, lesser local side effects/toxicity and increased bioavailability by increased drug eye contact time is observed [3, 4].

The eye is very difficult to study from a drug delivery point of view. The anatomy physiology and biochemistry of the eye render this organ impervious to foreign substances. It is a challenge to the formulator to circumvent the protective barriers of the eye so that the drug reaches the bio-phase in sufficient concentration [4]. Ocular inserts have been developed in which the drug is delivered on the basis of diffusion mechanism. Such a solid dosage form delivers an ophthalmic drug at near constant rate, minimizing side effect by avoiding absorption peaks [5].

\section{CONVENTIONAL OPHTHALMIC FORMULATIONS Eye Drop}

It is a liquid preparation in which all ingredients are completely dispersed in solution. Limited ocular absorption occurs through corneal epithelium. When eye drops is topically administered then only less than $5 \%$ of administered dose is absorbed. The retention time of eye solution in the eye can be influenced by various properties of eye solution like hydrogen ion, concentration, viscosity etc.

\section{Eye Gel}

It is a semi solid preparation made up of either small or larger molecules are interpenetrated by liquid. When this preparation is applied in the eye region, results in increased drug absorption, contact time and provides prolonged duration of action/therapeutic effect and decreased dosage frequency compare to solutions.

\section{Eye Ointment}

It is a sterile and semisolid anhydrous preparation in which medicament is dispersed in a suitable non-irritant bases like mineral oil and white petroleum. The eye ointment remains popular as a pediatric dosage form. The ointment provides longer contact time and increased drug bioavailability after application in the eye, but its use is limited to bed time 
instillation because it interferes with vision due to its greasy nature.

\section{Eye Suspension}

It is defined as the preparation in which the drug substances, insoluble in an aqueous vehicle, are dispersed with the help of a suitable suspending and dispersing agent. The eye suspensions contain small particles which are non-irritating to the eye. These particles are retained in cul-de-sac region and thus increased drug bioavailability results [10].

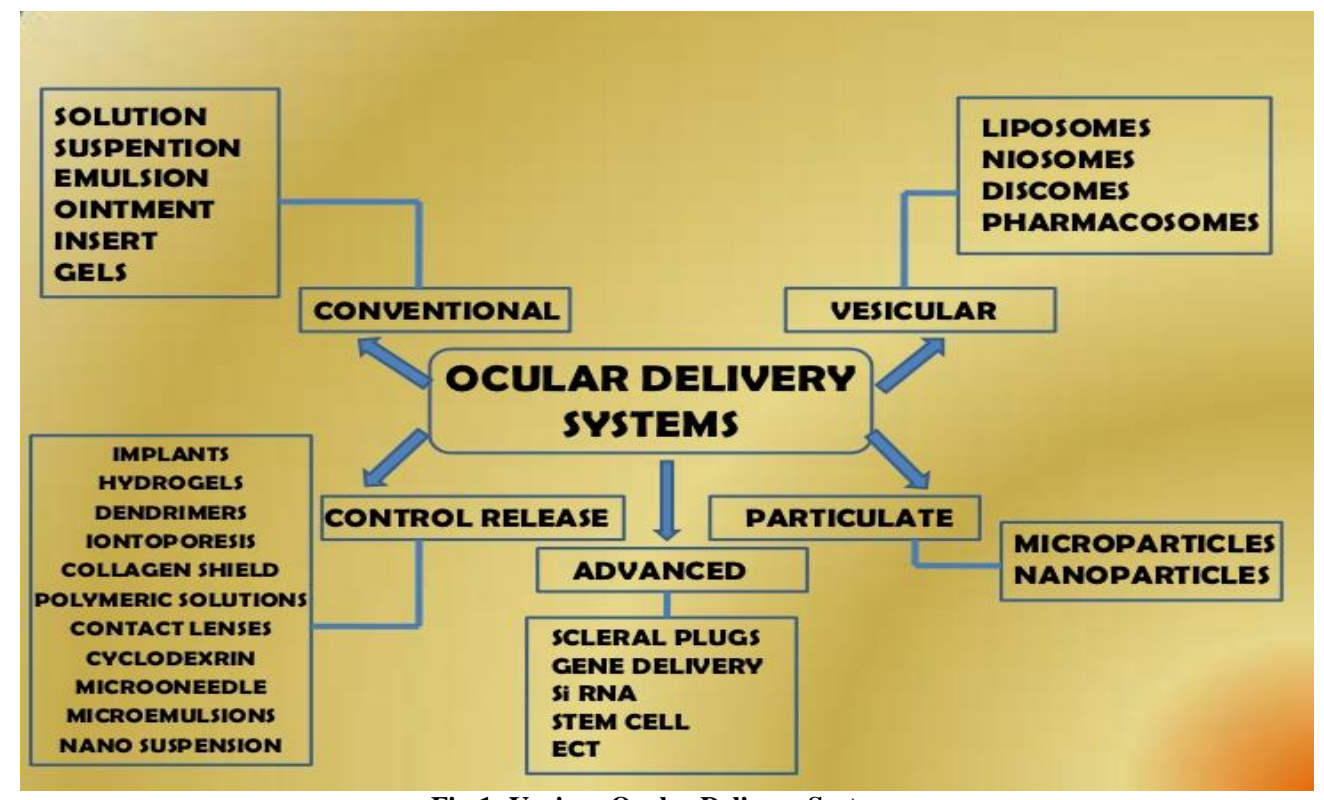

Fig-1: Various Ocular Delivery Systems

DRAWBACKS OF CONVENTIONAL OPHTHALMIC FORMULATION

- They have poor bioavailability due to:

$>$ Conjuctival absorption

$>$ Solution drainage by nasolachrimal route

$>$ Rapid pre-corneal elimination

$>$ Normal tear turnover

- Viscous vehicles may cause blurred vision.

- Frequent instillation is required/necessary to achieve a therapeutic effect of concentrated medicine.

- Produce undesirable side effects after instillation of ophthalmic formulations due to systemic absorption of drug and nasolachrimal drainage of additives.

- The delivery of drug is generally not correct due to non-uniformity of drop size of ocular medication [11].

\section{Novel Ophthalmic Delivery System}

To overcome the drawbacks of conventional ophthalmic formulations, various workups have been done to better pre-corneal drug absorption and minimizing pre-corneal drug elimination.

\section{Mucoadhesives}

Mucoadhesive is defined as the formulation which is retained in the eye by virtue of non-covalent bonds accomplished with the corneal conjuctival mucin for improving the pre-corneal residence time.

\section{Phase Transition Systems}

The phase transition system is a liquid dosage form which converts into the gel or solid phase when instilled into the cul-de-sac of eye. These converting gel remains in contact with the cornea of the eye for prolonged period of time due to slower drainage from the cornea.

\section{Liposomes}

Liposomes are defined as simple microscopic vesicles in which aqueous volume is entirely enclosed by a bilayered phospholipids membrane. The lipophilic drug is entrapped in bilayer of lipids and hydrophilic drug in aqueous phase and thus increased drug residence time in the eye is achieved.

\section{Niosomes}

Noisomes are non ionic surfactant small vesicles in which both hydrophilic and lipophilic drugs are enclosed in aqueous or in vesicular membrane, made up of lipid material. It prevents the metabolism of the drug by enzymes, present at the corneal surface of the eye.

\section{Nanoparticles}

Nanoparticles are solid particles made up of polymers with particle size ranging from 1-1000 nm in which the drug or active ingredient is entrapped. These entrapped drugs provide a sustained effect. 


\section{Contact Lenses}

The drug saturated contact lenses are placed in the eye which releases the drug in the eye for a prolonged period of time. Hydrophilic or water soluble drugs soaked in drug solution can be absorbed through contact lenses and thus hydrophilic contact lenses can be used for improving ocular residence time of drug.

\section{Pharmacosomes}

Pharmacosomes are vesicles made up of amphiphilic drugs. Any drug having a free carboxylic group or active hydrogen atom can be esterified to the amphiphilic prodrug. These amphiphilic pro-drugs are converted to pharmacosomes on dilution with water and thus show greater shelf stability and facilitated transport across the cornea and a controlled release profile [10, $11]$.

\section{Ophthalmic Inserts}

Ocusert system was firstly developed in 1975 by 'Alza Corporation, in the United State of America. It is a flat, flexible, solid and semisolid device which consists of drug reservoir and rate controlling membrane by using various polymers $[4,12]$. The prime objective of development of the ocuserts is continuous controlled delivery of ophthalmically active drug to the eye. The ocusert is inserted in the upper or lower cul-de-sac of the eye, which releases the drug at a predetermined rate constant. Thus improved patient compliance by reduced dose frequency, better therapeutic outcomes by reduced over/under dosing, lesser local side effects/toxicity and increased bioavailability by increased drug eye contact time is observed [13]. Generally, all types of ocusert consist of three components namely, "a central drug reservoir" in which the drug is incorporated in a polymer; "rate controlling membrane", which ensures the controlled release of medicament from the drug reservoir; and "an outer annular ring", meant for easy handling and proper insertion [8].

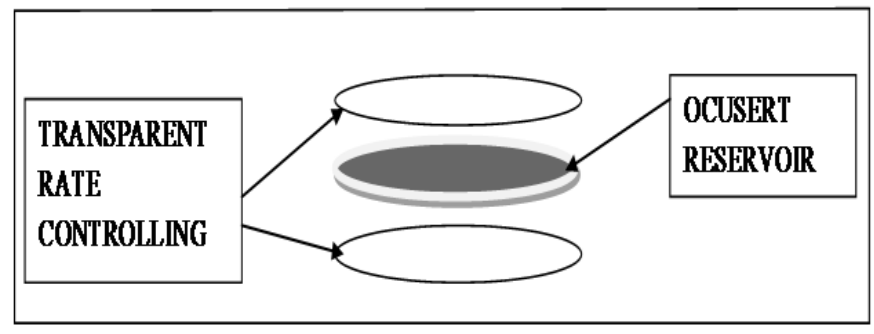

Fig-2: Schematic diagram of ocular insert

\section{Advantages of ocusert}

Ocusert enjoys following advantages over conventional ophthalmic dosage forms:-

1. Increased ocular contact time and thus improved drug bioavailability [11].

2. Increased ocular permeation with respect to standard formulation and thus providing prolong drug activity and hence increased ocular bioavailability of drug [13].

3. Administration of an accurate dose in the eye gives better therapy [11].

4. Better patient compliance by reduction of the number of administered dose [10].

5. Better efficacy by providing a constant drug release [11].
6. Increased possibility of internal ocular tissue targeting through non-corneal, (conjuctival and sclera) penetration routes.

7. Increased shelf life with respect to standard formulation due to the absence of water [13].

8. Reduced systemic absorption and thus lesser adverse effects.

\section{Limitations of Ocusert}

- Initially discomfort due to their movement around the eye [11].

- Occasional accidental loss during sleep or while rubbing the eye.

- Difficult placement and intervention with vision [10].
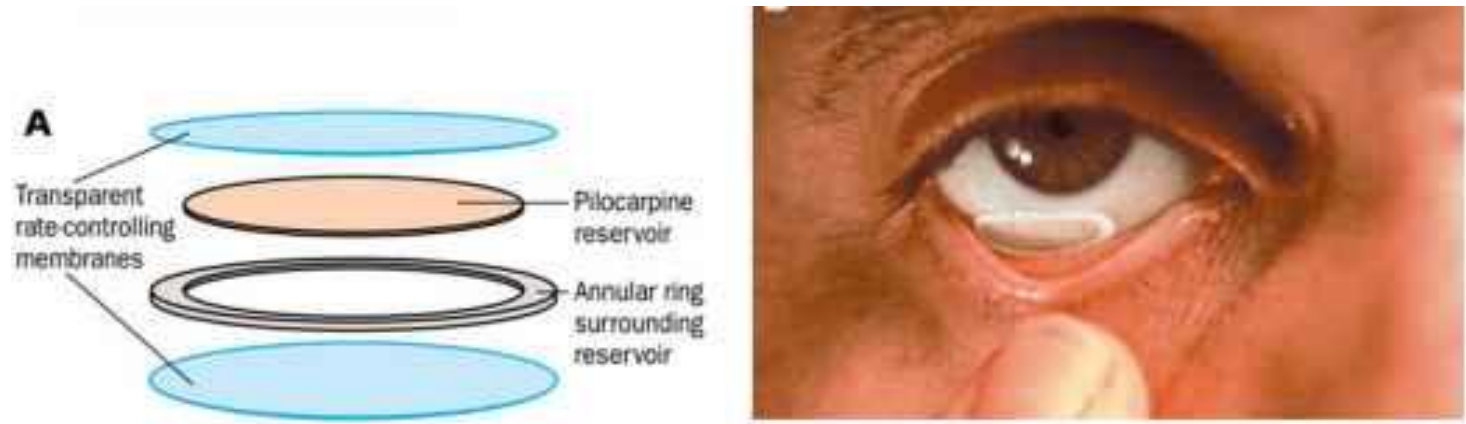

Fig-3: Delivery of drug via ocular insert 


\section{MECHANISM OF CONTROL DRUG RELEASE INTO} THE EYE

The mechanism of controlled drug release into the eye is as follows:-

- Diffusion

- Osmosis

- Bio-erosion

\section{Diffusion}

In the diffusion mechanism, the drug is released incessantly at a controlled rate through the membrane into the tear fluid. If the insert is consist of a solid non erodible body with pores and dispersed drug. The release of the drug occurs through the pores via diffusion. Controlled drug release can be further regulated by gradual dissolution of solid dispersed drug and thus this matrix as a result of directed diffusion of aqueous solutions. In a soluble device, true dissolution mainly occurs through polymer swelling. In swelling controlled devices, the active ingredient is homogeneously dispersed in a glassy polymer. These glassy polymers are essentially drug impermeable, no diffusion occurs through the dry matrix. When the ocular insert is inserted in the eye, the water from the tear fluids begins to penetrate the matrix, then swelling and thus polymer chain relaxation and drug diffusion occurs. The dissolution of the matrix follows the swelling process which depends on polymer structure [14].

\section{Osmosis}

In the osmosis mechanism, the insert consist of a transverse impermeable elastic membrane dividing the interior of the insert in to a first compartment and second compartment, the first compartment is bounded by a semi-permeable membrane and the impermeable elastic membrane, and the second compartment is bounded by impermeable and elastic membrane. There is a drug release aperture in the impermeable wall of insert. The first compartment contains a solute which cannot pass through the semi-permeable membrane and the second compartment provides a reservoir for the drug which a gain is in liquid or gel form. When the insert is inserted in the aqueous environment of the eye then water diffuse into the first compartment and stretches and contract the second compartment so that the drug is forced through the drug release aperture [15].

\section{Bio-Erosion}

In the Bio-erosion mechanism, the insert is made up from a matrix of bio-erodible material in which the drug is dispersed. When insert contact with tear fluid and gives results in controlled sustained release of the drug by bio-erosion of the matrix. The drug may be homogeneously dispersed throughout the matrix but it is believed a more controlled release is obtained if the drug is superficially concentrated in the matrix [16].

\section{CLASSIFICATION OF OPHTHALMIC INSERTS/OCUSERTS}

The ophthalmic inserts/ocuserts are classified on the basis of their solubility behavior.

1. Insoluble ophthalmic inserts/ocuserts

2. Soluble ophthalmic inserts/ocuserts

3. Bio-erodible ophthalmic inserts/ocuserts [10]

\section{Insoluble ophthalmic inserts/ocuserts} three groups

The insoluble inserts are sub-classified into
a) Diffusional inserts/ocuserts
b) Osmotic inserts/ocuserts
c) Contact lenses [17]

\section{Diffusional Inserts/Ocuserts}

Ocuserts system is a novel drug delivery system is based on porous membrane. The release of drug from diffusional inserts/ocuserts is based on a diffusional release mechanism. The diffusional inserts/ocuserts are consist of a central drug reservoir enclosed in specially designed semi permeable/microporous membrane, which allow the diffusion of drug from the reservoir at a precisely determine rate [14]. The lachrymal fluid penetrating through the membrane until the sufficient internal pressure is reached which control the drug release from such a system. The principle for its operation can be operated by the Fick diffusion equation.

$$
\mathbf{J}=\frac{\text { DA dc }}{\mathbf{d x}}
$$

Where,

$$
\begin{aligned}
& \mathbf{J}=\text { Solute flux } \\
& \mathbf{D}=\text { Difference co-efficient for the drug within } \\
& \text { the polymer membrane. } \\
& \mathbf{A}=\text { Area of membrane } \\
& \frac{\mathbf{d c}}{\mathbf{d} \mathbf{x}}=\text { Drug concentration gradient within the } \\
& \text { membrane along the direction } \\
& \text { of drug flow [10]. }
\end{aligned}
$$

\section{Osmotic Inserts/Ocuserts}

The osmotic inserts/ocusert are generally consist of two distinct contains osmotic solute. The drug and the osmotic solute are placed in to seprate compartment, the drug reservoir being surrounded by an elastic impermeable membrane and the osmotic solute reservoir by a semi-permeable membrane [14]. The tears diffusion in to the osmotic compartment inducing on osmotic pressure due to drug diffusion [10].

\section{Contact Lenses}

Contact lenses are covalently cross-linked hydrophilic or hydrophobic polymer that forms a three dimensional network which able of retaining aqueous drug solution or solid components ${ }^{(10)}$. Contact lenses are structures shaped and initially used for vision correction. Contact lenses are widely used as a potential drug delivery device by presoaking them in drug solutions. The main advantage of this system is increase 
the possibility of correct vision and drug release at the same time [14].

\section{Soluble Ophthalmic Inserts/Ocuserts}

Soluble ophthalmic inserts/ocuserts represent to the oldest class of ophthalmic inserts/ocuserts. These soluble inserts/ocuserts offer the advantage of completely soluble so that they do not need to be removed from their site of application thus, limiting the interference to insertion only. The therapeutic agents are preferably absorbed by soaking the insert in a solution containing the drug, dry and re-hydrating it before use on the eye. The amount of drug loaded will be depend upon the amount of binding agent, upon the concentration of drug solution in to which the composite is soaked as well as the duration of soaking (17). The release of drug from this type of inserts/ocuserts due to permeation of tear fluid into the inserts that increases high release rate of drug through diffusion and form a gel layer around the core of the insert [10].

\section{Bio-Erodible Inserts/Ocuserts}

The bio-erodible inserts/ocuserts are consists of material homogenous dispersion of drug included or not into a hydrophobic coating which is substantially impermeable [17]. The bio-erodible inserts/ocusert consist of bio-erodible poly (e.g. cross-linked gelatin derivatives, polyester derivative) which undergo hydrolysis of chemical bond and hence dissolution. The main advantage of these bio-erodible polymers is the possibility of modification of their erosion rate by modifying their final structure during synthesis and by addition of anionic or cationic surfactants [14]. The release of the drug from such a system is the outcomes of the contact of the device with the tear fluid inducing a superficial diversion of the matrix [10].

According to the world health organization, corneal disease is a major cause of loss of vision and blindness. The fungal keratitis is a serious corneal disease. The first line therapy in fungal keratitis are not effective in severe keratomycosis, while imidazole derivatives prepared themselves be the as a better choice in the future [18]. Clotrimazole is a synthetic broad spectrum an imidazole derivative antifungal agent effective against keratitis, pathogenic dermatophytes, yeasts, several species of candida, trichophyton, mycotics and tinea infections like ringworm [19]. Clotrimazole is a mostly and effectively used through topical route for fungal infection because oral use of Clotrimazole is unacceptable due to the severe side effect and plasma half life is 3-6 hours, thus topical administration is recommended/required because having half life only 2 hours [20]. Clotrimazole is practically insoluble in water and poorly absorbed from gastrointestinal tract and it is metabolized into inactive compound by hepatic metabolism. Hence, it is requisite to manipulate the drug delivery system of Clotrimazole for topical administration [21].

Table-1: List of ocuserts of different ophthalmic drugs (all categories) available in market

\begin{tabular}{|c|c|c|c|}
\hline $\begin{array}{l}\text { S. } \\
\text { No }\end{array}$ & Drug & Category of drug & Polymer/Bases \\
\hline 1 & Ciprofloxacin & $\begin{array}{l}\text { Anti-Infective } \\
\text { Agent }\end{array}$ & HPMC, MC, EC and PVP \\
\hline 2 & Ofloxacin & Antibacterial agent & Poly ethylene oxide and EUD L100 \\
\hline 3 & Ciprofloxacin & Anti-Infective agent & Eudragit and Polyvinyl acetate \\
\hline 4 & Norfloxacin & Antibacterial agent & HPMC, EC and PVP K-30 \\
\hline 5 & Pefloxacin & Antibiotic agent & EUD RS100, EUD RL100 and PVP K-30 \\
\hline 6 & Ofloxacin & Antibacterial agent & HPMC, PVP and PVA \\
\hline 7 & Acyclovir & Antiviral agent & MC, HPMC, HPC and Starch \\
\hline 8 & Acyclovir & Antiviral agent & PVA, MC, EC and Carbapol 934 \\
\hline 9 & Levofloxacin & Antibacterial agent & EC, PEO and sodium alginate \\
\hline 10 & Brimonidine Tartrate & Intraocular pressure lowering agent & PVA, EC and PVP K-30 \\
\hline 11 & Fluconazole & Antifungal agent & HPMC, PVP and PVA \\
\hline 12 & Natamycin & Polyene antibiotic agent & $\begin{array}{l}\text { EUD L100, EUD S100, EUD RL100, HPMC and } \\
\text { EC }\end{array}$ \\
\hline 13 & Indomethacin & Non-steroidal anti-inflammatory agent & EUD L100, EUD RL100, HPMC and EC \\
\hline 14 & Levobunolol & $\beta$-blocker agent & MC, PVP and HPMC \\
\hline 15 & Levobunolol & $\beta$-blocker agent & EC and EUD RL100 \\
\hline 16 & Timolol Maleate & Anti-glaucoma agent & $\begin{array}{l}\text { MC, HPC, EUD RL100, EUD RS100, EC and } \\
\text { PVP }\end{array}$ \\
\hline 17 & Aceclofenac & Non-steroidal anti-inflammatory agent & HPMC and EC \\
\hline 18 & Moxifloxacin & Antibacterial agent & EUD RL100, EUD RS100 and NaCMC \\
\hline 19 & $\begin{array}{l}\text { Ketorolac } \\
\text { Tromethamine }\end{array}$ & Non-steroidal anti-inflammatory agent & Gelatin, HPMC and EC \\
\hline 20 & Natamycin & Polyene antibiotic agent & MC, Sodium alginate and Gelatin \\
\hline 21 & Diclofenac Sodium & Non-steroidal anti-inflammatory agent & HPMC, and EUD L100 \\
\hline 22 & Moxifloxacin & Antibacterial agent & Chitosan, MC and HPMC K4M \\
\hline
\end{tabular}




\section{CONCLUSION}

The ocular insert represents a significant advancement in the therapy of eye disease. Ocular inserts are characterized as clean, thin, multilayered, medicate impregnated, strong or semisolid consistency gadgets set into the cul-de-sac or conjuctival sac, whose size and shape are particularly intended for ophthalmic application. They are made out of a polymeric bolster that might possibly contain a medication. Expanding contact time and along these lines enhancing bioavailability. Conceivable diminishment of systemic absorption and in this manner lessened systemic antagonistic impacts. Lessened recurrence of organizations and therefore better patient consistence with lower occurrence of visual side effects. In this survey, we have focused on the advanced approaches in ocular drug delivery system. Advantages with ocuserts such as, Accurate dosing Capacity to provide at constant rate and prolong drug release thus a better efficacy. Increasing contact time and thus improving bioavailability. Possible reduction of systemic absorption and thus reduced systemic adverse effects. Reduced frequency of administrations and thus better patient compliance with lower incidence of visual side effects.

\section{REFERENCES}

1. Sachdeva, D., \& Bhandari, A. (2011). Design, formulation, evaluation of levobunolol $\mathrm{HCl}$ ocular inserts. Journal of Pharmaceutical Sciences and Research, 3(12), 1625-1631.

2. Paswan, S. K., Verma, P., \& Yadav, M. S. (2015). Review-Advance Technique in Ocular drug delivery system. World journal of Pharmacy and Pharmaceutical sciences, 4, 346-365.

3. Sreenivas, S. A., Hiremath, S. P., \& Godbole, A. M. (2006). Ofloxacin ocular inserts: Design, formulation and evaluation. Iranian Journal of Pharmacology and Therapeutics, 5(2), 159-162.

4. Jain, N. K. (2009). Controlled and novel drug delivery", First Edition 1997, Reprint 2009, CBS publishers and distributors, 11 Darya Ganj, New Delhi-110002 (India), 82-89.

5. Beringer, P. (2009). "Remington the science and practice of pharmacy", $21^{\text {st }}$ edition 2005 , third Indian reprint 2009, Wolter kulwer publication (India) Pvt. Ltd., New Delhi, 850-862.

6. Vishal, P., \& Agrawal, Y. K. (2011). Current status and advanced approaches in ocular drug delivery system. Journal of global trends in pharmaceutical sciences, 2(2), 131-148.

7. Cheien, Y. W. "Drugs and the pharmaceutical sciences novel drug delivery systems", Second edition, revised and expanded, volume 50, Informa health care USA inc. 52 Vanderbit avenue New York NY 10017, 269-270.
8. Thakur, R., \& Swami, G. (2012). Promising implication of ocuserts in ocular disease. Journal of Drug Delivery and Therapeutics, 2(2):18-19.

9. El Gamal, S. S., Naggar, V. F., \& Allam, A. N. (2008). Formulation and evaluation of acyclovir ophthalmic inserts. Asian J Pharm Sci, 3(2), 5867.

10. Imam, S., Bansal, A., Bushetti, S. S., Singh, A., \& Chopra, H. (2009). Novel ocular dosage form in the treatment of glaucoma. The Pharma Research, Sudarshan Publication, 1, 72-81.

11. Patel, J. K., \& Gevariya, M. H. B. (2013). Formulation and evaluation of sustained release ocular drug delivery system for an anti-glaucoma drug. Saurashtra University, 1-21.

12. Bankar, G. S., \& Rhodes, C. T. Drug and the pharmaceutical sciences modern pharmaceutics, Second edition, revised and expanded, Volume 40, Marcel Daker, inc., 270 Madison Avenue, New York, New York 10016, 573-574.

13. Brahmankar, D. M., \& Jaiswal, S. B. (2010). Biopharmaceutics and pharmacokinetics a treatise, second edition, reprint 2010, Vallabh prakashan New Delhi-110088; 473.

14. Kumar, B. P., Harish, G., \& Bhowmik, D. (2013). Ocular inserts: A novel controlled drug delivery system. The Pharma Innovation, 1(12):1-14.

15. Karthikeyam, D. (2008). The concept of ocular inserts as drug delivery systems: An overview, AJP. 194-196.

16. Dabhi, V., Yogi, J., Bhimani, B., Patel, U., \& Patel, G. (2014). Ocular inserts as controlled drug delivery systems. International Journal of Pharmaceutical Research and Bio-Science, 3(5), 468-480.

17. Rajesh, A., Poonam, D., \& Sangeeta, A. (2012). Ocular Drug Delivery System: Ocular Insert. International Journal of Universal Pharmacy and Bio Sciences, 1(2):30-38.

18. Rasool, B. K. A., \& Salmo, H. M. (2012). Development and Clinical Evaluation of Clotrimazole- $\beta$-Cyclodextrin Eyedrops for the Treatment of Fungal Keratitis. AAPS PharmSciTech, 13(3), 883-889.

19. Hashem, F. M., Shaker, D. S., Ghorab, M. K., Nasr, M., \& Ismail, A. (2011). Formulation, characterization, and clinical evaluation of microemulsion containing clotrimazole for topical delivery. AAPS PharmSciTech, 12(3), 879-886.

20. Ning, M., Gu, Z., Pan, H., Yu, H., \& Xiao, K. (2005). Preparation and in vitro evaluation of liposomal/niosomal delivery systems for antifungal drug clotrimazole.

21. Shirsand, S. B., Kumar, G. R., Keshavshetti, G G., Bushetti, S. S., \& Swamy, P. V. (2015). Formulation and evaluation of clotrimazole niosomal gel for topical application. RGUHS $J$ Pharm Sci, 5(1), 32-38. 